\title{
Neutrophil extracellular trap formation is regulated by CXCR2 in COPD neutrophils
}

\author{
To the Editor:
}

Chronic obstructive pulmonary disease (COPD) is characterised by persistent neutrophilic inflammation of the airways, which is associated with enhanced neutrophil extracellular trap (NET) formation of airway neutrophils [1-3]. NETs are web-like extracellular structures consisting of neutrophil DNA components and degradative enzymes (e.g. neutrophil elastase and myeloperoxidase) which are released from azurophilic granules by activated neutrophils [4]. Initially found to immunoregulate host defence responses during bacterial infections, emerging data indicate that exaggerated NET formation and/or delayed NET clearance may inflict tissue damage and organ dysfunction in a range of human inflammatory diseases independent of infections [5, 6]. Recently, we found NET formation of sputum neutrophils and extracellular DNA levels in sputum supernatant to be significantly enhanced in patients with stable COPD irrespective of the current smoking state or the purulence of the sputum samples [1]. Several studies, including our own, show that the amount of NET formation and/or level of extracellular DNA in patients with stable COPD correlate with the airflow limitation, suggesting that NET formation by airway neutrophils may contribute to the lung tissue damage observed in COPD [1-3]. However, the underlying mechanisms inducing NET formation in COPD neutrophils are largely unexplored. Furthermore, it is unknown whether neutrophils undergo NETosis following migration into the lung tissues or whether neutrophils are constitutively poised to undergo this response in circulation during COPD-related inflammation. It is notable that there is an increase in the release of cell-free DNA and myeloperoxidase that are active constituents of NETs in peripheral blood in COPD patients [7-9]. Here, we aimed to compare NET formation in autologous neutrophils derived from peripheral blood and sputum from COPD patients ex vivo. We also investigated whether the selective CXCR2 antagonist AZD5069, that has been shown to block trafficking of neutrophils from blood into airways in bronchiectasis patients [10] and more recently in a small cohort of neutrophilic asthma patients [11], could dampen NET formation in COPD-derived neutrophils ex vivo.

We evaluated blood and induced sputum samples from 12 COPD patients: seven smokers and five ex-smokers; mean age $67.8 \pm 7.5$ years; mean forced expiratory volume in $1 \mathrm{~s} 59.6 \pm 12.4 \%$ pred; mean pack years of smoking $61.8 \pm 20.5$ years; no treatment with inhaled corticosteroids or roflumilast; no exacerbation or respiratory tract infection within 6 weeks prior to the study visit. This study was approved by the Ethics Committee [144/15] and all patients gave written informed consent. Peripheral blood (24 mL) was drawn from COPD patients and neutrophils were isolated according to BRINKMANN et al. [12]. Induced sputum from the same COPD patients was collected, processed and analysed as previously described [1]. Approximately $1 \times 10^{6}$ neutrophils harvested from sputum and blood were seeded on poly-D-lysine coated glass coverslips in triplicate. To assess the effects of AZD5069 on NET formation, cells were incubated with $100 \mu \mathrm{M}$ AZD5069 for $1 \mathrm{~h}$ at $37^{\circ} \mathrm{C}$ in air, 5\% CO $\mathrm{CO}_{2}$. NET formation of blood neutrophils ex vivo was stimulated by incubation with sputum supernatant derived from the same COPD patient in an autologous manner $\left(2 \mathrm{~h}, 37^{\circ} \mathrm{C}\right.$ in air, $\left.5 \% \mathrm{CO}_{2}\right)$. The potential effects of $0.1 \%$ dithiothreitol present in sputum supernatant on inducing NET formation was assessed, and no discernible effects were observed (data not shown). NETs were quantified using ImageJ software (National Institutes of Health, Bethesda, MD, USA) by marking NETs as regions of interest (ROI) as reported previously [1]. Concentration of extracellular DNA in cell culture medium was quantified using a NanoDrop 2000 spectrophotometer (ThermoFischer Scientific, Wilmington, DE, USA). Chemokine CXCL8 levels in cell culture fluid were quantified by ELISA (R\&D systems, Abingdon, UK). The maximally effective concentration of AZD5069 (100 $\mu \mathrm{M})$ in blocking

@ERSpublications

CXCR2 might have a crucial role in neutrophil extracellular trap formation in COPD neutrophils http://ow.ly/LRst30iE1Bw

Cite this article as: Pedersen F, Waschki B, Marwitz S, et al. Neutrophil extracellular trap formation is regulated by CXCR2 in COPD neutrophils. Eur Respir J 2018; 51: 1700970 [https://doi.org/10.1183/ 13993003.00970-2017]. 
a)
DNA (DAPI)
Histone H1 (Alexa 549)

NE (Alexa 549)
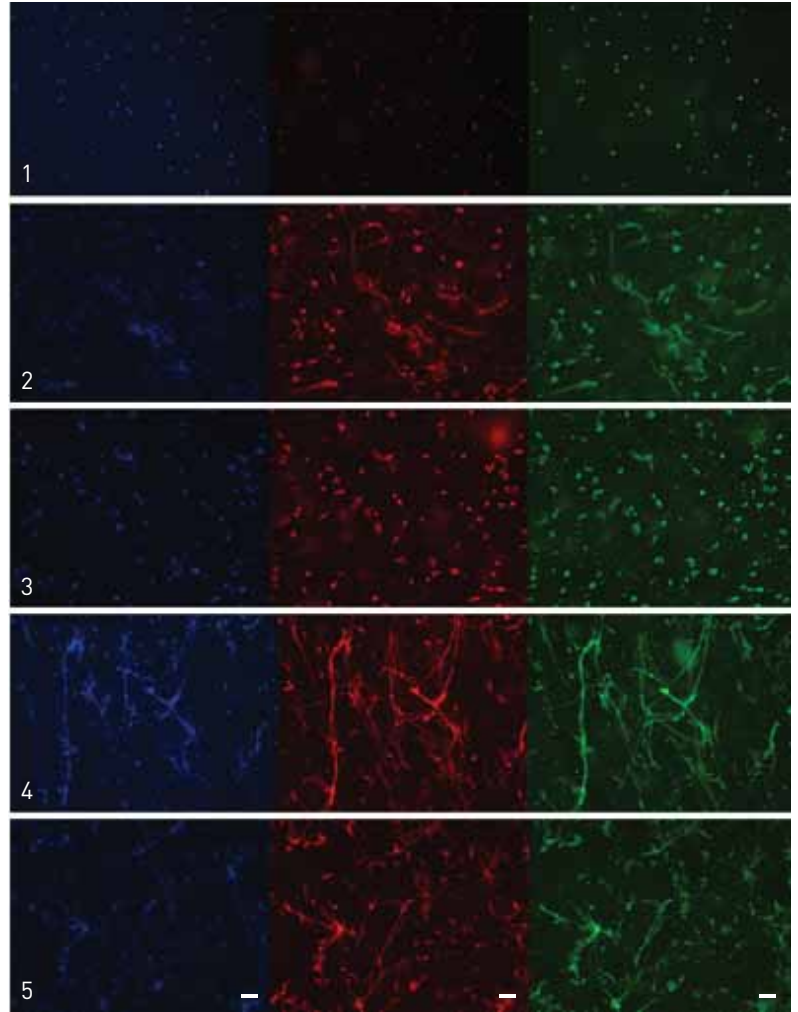

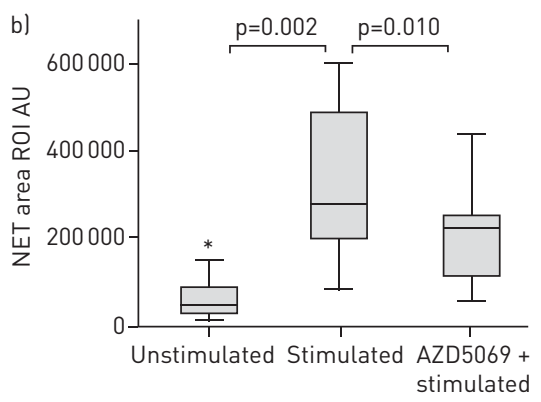

d)
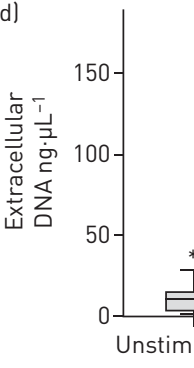

$p=0.008$

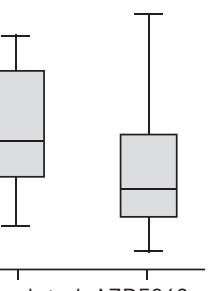

f)

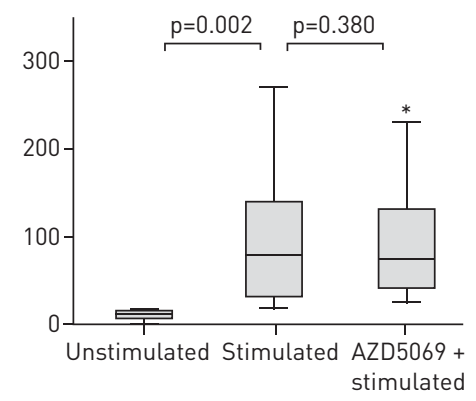

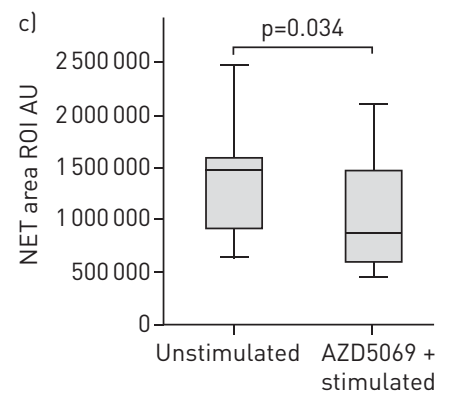
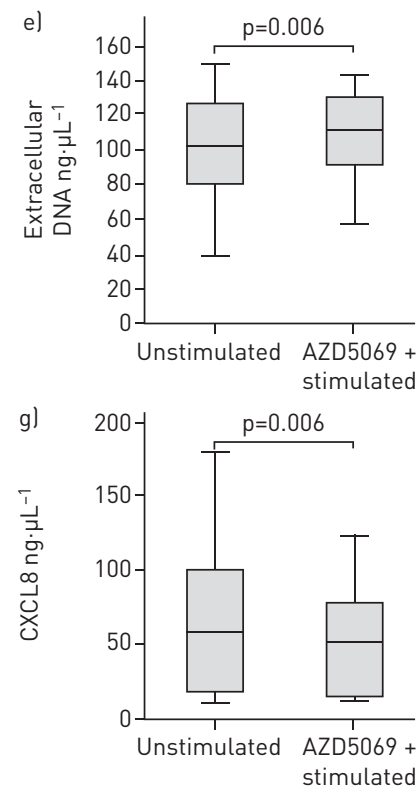

FIGURE 1 CXCR2 regulates neutrophil extracellular trap (NET) formation in chronic obstructive pulmonary disease (COPD) in vitro. a) Representative fluorescence images of NET formation in neutrophils derived from COPD patients. All specimens were fixed in phosphate buffered $4 \%$ paraformaldehyde. Immunofluorescence staining for detection of histone H1, neutrophil elastase (NE) and DNA with 4,6-diamidino-2-phenylindole (DAPI) was performed as described previously [1]. Representative visual fields of all specimens were visualised (Nikon ECLIPSE 80i fluorescence microscope, Tokyo, Japan) and photographed (QImaging QCam FAST 1394, Surrey, BC, Canada) to digitally capture H1-/NE-/DNA-stained images using identical exposure times. Row 1: unstimulated blood neutrophils; row 2: blood neutrophils stimulated by sputum supernatant; row 3 : blood neutrophils stimulated by sputum supernatant after pretreatment with 100 $\mu$ M AZD5069; row 4: unstimulated sputum neutrophils; row 5: unstimulated sputum neutrophils after pretreatment with $100 \mu M$ AZD5069. Original magnification $\times 100$. Quantification of NET areas in b) cultivated blood and c) sputum neutrophils. CXCR2 antagonist AZD5069 reduces NET areas of blood neutrophils stimulated by autologous COPD sputum supernatant. All assessments were performed three times and the intra-individual mean value was used for the analyses. Extracellular DNA concentration in d) cultivated blood neutrophils and e) sputum neutrophils. CXCR2 antagonist AZD5069 reduces DNA release by blood neutrophils stimulated by autologous COPD sputum supernatant. Extracellular DNA concentrations were also measured three times and the intra-individual mean value was used for the analyses. CXCL8 concentration in f) cultivated blood neutrophils and gl sputum neutrophils. CXCL8 concentrations were determined twice in each subject and the mean value was used for further analyses. ROI: region of interest. *: outlier.

NET formation in blood neutrophils was obtained in pilot dose-titrating experiments in vitro (data not shown). Statistical analyses were performed using SPSS version 20 (SPSS Inc; Chicago, IL, USA). Variables of interest (i.e. ROI, DNA and CXCL8) were skewed and presented as median (interquartile range). Differences between groups were analysed by Wilcoxon matched-pairs signed-rank test. $\mathrm{p}<0.05$ was considered statistically significant.

Purity and viability of isolated blood neutrophils was $98.9 \pm 1.0 \%$ and $98.3 \pm 2.2 \%$, respectively. Neutrophil purity in homogenised sputum samples was $84.3 \pm 12.4 \%$ with a viability of $89.7 \pm 8.4 \%$. Representative photomicrographs illustrating NET formation in isolated blood and sputum neutrophils are shown (figure 1a). Spontaneous NET formation was absent in peripheral blood neutrophils after incubation for $2 \mathrm{~h}$ at $37^{\circ} \mathrm{C}$ in air, $5 \% \mathrm{CO}_{2}$ (figure 1a row 1). However, upon stimulation with sputum supernatant of the same individual, significant NET formation was observed (figure 1a row 2). Compared to naïve, unstimulated blood neutrophils, NET formation was significantly increased $(p=0.002)$ by five-fold when blood neutrophils were stimulated with sputum supernatant (figure 1b). Extracellular DNA concentration was approximately eight-fold higher and statistically significant $(p=0.003)$ in stimulated blood neutrophils 
compared to unstimulated blood neutrophils (figure 1d). CXCL8 concentration in stimulated blood neutrophil cell culture fluid was significantly higher $(\mathrm{p}=0.002)$ than in unstimulated blood neutrophils (figure 1f). The effects of AZD5069 $(100 \mu \mathrm{M})$ treatment were assessed in stimulated blood neutrophils. Compared to vehicle, CXCR2 antagonism significantly reduced $(p=0.010)$ NET formation in blood neutrophils (figure 1b). Treatment with AZD5069 led to significant decreases $(p=0.006)$ in extracellular DNA concentration in stimulated neutrophils relative to vehicle-treated cells (figure 1d). Antagonism of CXCR2 with AZD5069 had no significant effect ( $\mathrm{p}=0.388$ ) in CXCL8 concentration in cell culture fluid (figure 1f).

Sputum neutrophils showed spontaneous NET formation and this response was significantly decreased $(\mathrm{p}=0.034)$ in the presence of AZD5069 (figure 1a (rows 4 and 5) and figure 1c). In contrast, treatment with AZD5069 induced significantly increased $(\mathrm{p}=0.006)$ DNA concentrations in sputum cell culture medium relative to vehicle-treated sputum neutrophils (figure 1e). CXCL8 concentration in sputum cell culture medium was significantly lower $(\mathrm{p}=0.006)$ in the presence of AZD5069 (figure 1g).

In this translational study, we analysed NET formation, extracellular DNA and CXCL8 concentration in the presence of CXCR2 antagonism using AZD5069 in neutrophils derived from sputum and peripheral blood from COPD patients. To our knowledge, this is the first study to compare the levels of NET formation in autologous blood and sputum neutrophils of COPD patients simultaneously. We showed that blood neutrophils underwent NETosis upon stimulation by sputum supernatant, representing an inflammatory microenvironment conducive to the COPD airways [1-3]. Consistent with the CXCR2 antagonising effects of blocking neutrophil recruitment to the inflamed airways [11], the induced NET formation of blood neutrophils could be significantly reduced by AZD5069 treatment. This suggests that CXCR2 has an essential role in regulating NETosis via CXCL8-mediated mechanisms in blood-derived neutrophils from COPD patients. The regulatory role of CXCR2 signalling in NETosis in healthy subjects remains to be elucidated.

By contrast, the effects of CXCR2 antagonism on spontaneous NETosis of sputum neutrophils were less pronounced, suggesting that in neutrophils that may have already undergone NETosis, subsequent reversal of this cellular process via CXCR2 antagonism cannot be readily achieved. This would also explain why we observed partial effects of CXCR2 antagonism on extracellular DNA levels in cell culture during spontaneous NETosis of sputum neutrophils.

We conclude that neutrophils from patients with COPD are likely to undergo NETosis upon exposure to inflammatory microenvironments conducive to the COPD airways and that the chemokine receptor CXCR2 plays an essential regulatory role in NET formation in COPD neutrophils.

Frauke Pedersen ${ }^{1,2}$, Benjamin Waschki $^{2}$, Sebastian Marwitz ${ }^{3}$, Torsten Goldmann $\oplus^{3}$, Anne Kirsten ${ }^{1}$, Anna Malmgren ${ }^{4}$, Klaus F. Rabe ${ }^{2}$, Mohib Uddin ${ }^{4,5}$ and Henrik Watz ${ }^{1,5}$

${ }^{1}$ Pulmonary Research Institute at LungenClinic Grosshansdorf, Airway Research Center North (ARCN), German Center for Lung Research (DZL), Grosshansdorf, Germany. ${ }^{2}$ LungenClinic Grosshansdorf, Airway Research Center North (ARCN), German Center for Lung Research (DZL), Grosshansdorf, Germany. ${ }^{3}$ Research Center Borstel, Airway Research Center North (ARCN), German Center for Lung Research (DZL), Borstel, Germany. ${ }^{4}$ Respiratory, Inflammation and Autoimmunity IMED Biotech Unit, AstraZeneca, Gothenburg, Sweden. ${ }^{5}$ Both authors contributed equally.

Correspondence: Frauke Pedersen, Pulmonary Research Institute at LungenClinic Grosshansdorf, Woehrendamm 80, 22927 Grosshansdorf, Germany. E-mail: f.pedersen@pulmoresearch.de

Received: May 112017 | Accepted after revision: Jan 092018

Conflict of interest: F. Pedersen reports grants from AstraZeneca. B. Waschki reports grants from AstraZeneca. A. Kirsten reports grants from AstraZeneca, and personal fees from AstraZeneca and Boehringer Ingelheim. A. Malmgren is an employee of AstraZeneca. K. Rabe reports grants from AstraZeneca, and personal fees from AstraZeneca, BerlinChemie, Boehringer Ingelheim, Chiesi, Novartis, SanofiAventis, and Teva. M. Uddin is an employee of AstraZeneca. H. Watz reports grants from AstraZeneca, personal fees and non-financial support from AstraZeneca, GlaxoSmithKline and Novartis, and personal fees from BerlinChemie, Boehringer Ingelheim and Chiesi.

\section{References}

1 Pedersen F, Marwitz S, Holz O, et al. Neutrophil extracellular trap formation and extracellular DNA in sputum of stable COPD patients. Respir Med 2015; 109: 1360-1362.

2 Grabcanovic-Musija F, Obermayer A, Stoiber W, et al. Neutrophil extracellular trap (NET) formation characterises stable and exacerbated COPD and correlates with airflow limitation. Respir Res 2015; 16: 59.

3 Wright TK, Gibson PG, Simpson JL, et al. Neutrophil extracellular traps are associated with inflammation in chronic airway disease. Respirology 2016; 21: 467-475.

4 Brinkmann V, Reichard U, Goosmann C, et al. Neutrophil extracellular traps kill bacteria. Science 2004; 303: 1532-1535. 
5 Kessenbrock K, Krumbholz M, Schonermarck U, et al. Netting neutrophils in autoimmune small-vessel vasculitis. Nat Med 2009; 15: 623-625.

6 Khandpur R, Carmona-Rivera C, Vivekanandan-Giri A, et al. NETs are a source of citrullinated autoantigens and stimulate inflammatory responses in rheumatoid arthritis. Sci Transl Med 2013; 5: 178 ra40.

7 Cockayne DA, Cheng DT, Waschki B, et al. Systemic biomarkers of neutrophilic inflammation, tissue injury and repair in COPD patients with differing levels of disease severity. PLoS One 2012; 7: e38629.

8 Holz O, Roepcke S, Watz H, et al. Constant-load exercise decreases the serum concentration of myeloperoxidase in healthy smokers and smokers with COPD. Int J Chron Obstruct Pulmon Dis 2015; 10: 1393-1402.

9 Wielscher M, Vierlinger K, Kegler U, et al. Diagnostic performance of plasma DNA methylation profiles in lung cancer, pulmonary fibrosis and COPD. EBio Med 2015; 2: 929-936.

10 De Soyza A, Pavord I, Elborn JS, et al. A randomised, placebo-controlled study of the CXCR2 antagonist AZD5069 in bronchiectasis. Eur Respir J 2015; 46: 1021-1032.

11 Watz H, Uddin M, Pedersen F, et al. Effects of the CXCR2 antagonist AZD5069 on lung neutrophil recruitment in asthma. Pulm Pharmacol Ther 2017; 45: 121-123.

12 Brinkmann V, Laube B, Abu AU, et al. Neutrophil extracellular traps: how to generate and visualize them. J Vis Exp 2010; 36: 1724. 